\title{
LA DEMOCRACIA SUBYUGADA: EL HIPERPRESIDENCIALISMO VENEZOLANO
}

\author{
Venezuelan's Hiperpresidentialism: Democracy Subjugated
}

\section{MICHAEL PENFOLD}

Instituto de Estudios Superiores de Administración (Caracas, Venezuela)

\begin{abstract}
RESUMEN
El estado de la democracia en Venezuela combina prácticas electorales débilmente institucionalizadas aunque relativamente libres y limpias pero con claros rasgos autoritarios: la falta de autonomía de los poderes Judicial y Legislativo frente a un Ejecutivo con atribuciones extraordinarias; el desarrollo de complejas dinámicas de centralización del poder hacia la figura del Presidente; el acceso irrestricto a los recursos petroleros por parte del gobierno federal; la ausencia de una prensa imparcial e independiente y la pérdida de transparencia en el gobierno, entre otros. Las anormalidades constitucionales en el referéndum de 2009 (que permite el surgimiento del único sistema presidencial en la región con reelección ilimitada) sugieren además que incluso la democracia electoral está siendo socavada.
\end{abstract}

Palabras clave: Venezuela, democracia, presidencialismo, referéndum, descentralización.

\begin{abstract}
The state of democracy in Venezuela combines relatively fair, free, and electoral procedures but with distinct authoritarian features: the lack of autonomy in the Judicial and Legislative powers vis-à-vis an Executive with extraordinary attributions; the development of complex dynamics of centralization of power by the President; the free access to oil revenues by the federal government; the absence of an independent and impartial press and the loss of transparency in the government, among others. Moreover, the abnormalities in the 2009 referendum (that has allowed the emergence of the only presidential system in the region without term limits) suggest that even electoral democracy is being undermined in the country.
\end{abstract}

Key words: Venezuela, democracy, presidentialism, referendum, decentralization. 


\section{INTRODUCCIÓN}

La polarización política entre chavistas y antichavistas en Venezuela ha impactado todas las esferas de la vida social del país, al punto de incluso impedir por momentos una discusión académica sopesada de este importante fenómeno. Uno de los elementos más enigmáticos y divisivos en el mundo intelectual latinoamericano ha sido el debate entre distintos autores sobre la manera de caracterizar el sistema político venezolano, en particular, si el régimen chavista es de carácter autoritario o democrático. Los que defienden el corte democrático de la Venezuela contemporánea señalan que el país ha realizado desde 1998 más de ocho procesos electorales, que no sólo incluyen elecciones presidenciales, legislativas, regionales y locales sino también diversas formas de consultas populares (referéndums). Muchos autores señalan el carácter inclusivo de los programas sociales como una demostración de la vocación democrática, popular y progresista de este proceso político (Weisbrot y Sandoval, 2007; Fernandes, 2007). Por el contrario, otros autores han sostenido que el chavismo representa una nueva forma de autoritarismo latinoamericano que ha aprovechado el uso de nuevas tecnologías y el alto ingreso petrolero para tomar ventaja de las formas democráticas y minar de manera permanente la división de poderes (Corrales, 2006). Este debate ha empujado a varios autores a acuñar nuevos términos para etiquetar este régimen: "semiautoritarismo", "sultanismo" y "autoritarismo electoral" (Kornblith, 2007; Ottaway, 2003; Lupi y Vivas, 2005), con la intención de encajar el sistema político venezolano dentro de una tipología que permita caracterizar la paradoja que existe entre la existencia de elecciones competitivas y los rasgos autoritarios del proceso.

Este trabajo intenta situar dicho debate en una perspectiva que se adhiera a la teoría democrática. Para ello se analiza el funcionamiento del sistema político venezolano partiendo de la suposición de que efectivamente se trata de una democracia, con el propósito de entender de manera precisa las debilidades institucionales que influyen en la materialización de sus prácticas más relevantes. Como veremos, este análisis permite explicar con mayor exactitud las causas institucionales que puedan interferir en la consolidación del sistema democrático en Venezuela y producir su potencial colapso.

\section{CARACTERIZACIÓN DE LA DEMOCRACIA VENEZOLANA}

Esta sección pretende realizar una caracterización del funcionamiento actual de la democracia venezolana con respecto a tres dimensiones: la democracia electoral, la democracia constitucional y la democracia ciudadana. La democracia electoral se refiere a la capacidad de los votantes de ejercer su derecho al voto y escoger a sus candidatos libremente en elecciones competitivas (Dahl, 1980; Przeworski, 1991; Mainwaring, Brinks y Pérez Linán, 2001). La democracia constitucional se refiere al control popular sobre el ejercicio del poder por medio de la existencia de un estado de derecho caracterizado por la división de poderes. La democracia ciudadana es conceptualizada como los mecanismos y espacios disponibles para que los individuos puedan participar en las deliberaciones y decisiones colectivas que los afectan ( $\mathrm{O}^{\prime}$ Donnell et al., 2004). Esta última dimensión 
de la democracia también se refiere a la capacidad que tienen los individuos de ejercer sus derechos económicos y sociales para aumentar la efectividad de su participación política (Diamond y Morlino, 2004).

\section{a. La democracia electoral}

En Venezuela el derecho al voto está garantizado tanto en la Constitución Bolivariana como en la Ley Orgánica del Sufragio, para todos los ciudadanos mayores de 18 años sin exclusión alguna en términos de género, clase social o nivel educativo. Durante todos los procesos electorales bajo la presidencia de Hugo Chávez Frías y desde que fue aprobada la Constitución por la vía de un referéndum popular en diciembre de 1999, los venezolanos han tenido la posibilidad de ejercer el derecho al voto, incluso en ambientes de extrema polarización política.

El Consejo Nacional Electoral (CNE) ha intentado salvaguardar este derecho ampliando de forma significativa el padrón electoral, lo cual ha sido una fuente de preocupación entre los movimientos políticos adversarios al Presidente Chávez. Para poder registrarse como votantes, los venezolanos mayores de 18 años necesitan mostrar al CNE su identificación ciudadana vigente y proveer información sobre su lugar de residencia. La cédula no es expedida por el CNE sino por el Ministerio de Interior y Justicia a través de la Oficina de Identificación y Extranjería (ONIDEX). El gobierno nacional llevó a cabo entre 2003 y 2004 un proceso de cedulación y nacionalización masiva de ciudadanos a través de una campaña denominada Misión Identidad. Esta campaña fue en especial intensiva en los sectores de bajos ingresos de las zonas rurales y urbanas. El CNE aprovechó esta campaña para incluir a los nuevos ciudadanos en el Registro Electoral Permanente (REP) por medio de una coordinación administrativa con el Ministerio de Interior y Justicia para la implementación de la Misión Identidad (Penfold y Ortega, 2007).

La oposición observó esta campaña con preocupación debido al aumento súbito del número de votantes en el registro electoral poco antes del referéndum revocatorio del Presidente de la República en agosto de 2004. El registro electoral entre agosto y abril de 2004 creció en 11,7\% (Corrales y Penfold, 2007). Especialistas del Centro Carter verificaron después de una amplia revisión del padrón que el crecimiento del mismo no fue necesariamente excepcional. A pesar de ello, algunas organizaciones no gubernamentales especializadas en temas electorales aducían que era necesario auditar el REP, pues podía haber sido adulterado por medio de la Misión Identidad. Estas organizaciones esgrimían que una porción considerable de los votantes no podía ser adecuadamente identificada pues se carecía de datos sobre estos individuos, por ejemplo, la información exacta de su residencia. En el año 2006, el CNE accedió a una auditoría del REP por parte del Centro de Auditoría y Promoción Electoral (CAPEL). El informe de CAPEL validó la integridad del REP e identificó unas pequeñas fallas que no eran estadísticamente significativas, entre ellas, la repetición de algunos votantes y la necesidad de extraer difuntos del registro electoral (CAPEL, 2005). Ese mismo año, un conjunto de universidades nacionales auditó el REP con la autorización del CNE y reportó resultados similares. 
La transparencia y libertad en el ejercicio electoral es un tema más complejo de identificar que el ejercicio del derecho al voto. Esto se debe a que la conformación del CNE está dominada por el oficialismo con una representación minoritaria de la oposición (Kornblith, 2007). En principio, el CNE ha permitido que las elecciones sean automatizadas por completo, con la intención, en teoría, de impulsar la transparencia de los procesos electorales. Sin embargo, alrededor de este proceso ha habido muchas dudas con respecto a su implementación por parte de la oposición. El uso de dispositivos dactilares para la identificación de los votantes como requisito previo para votar fue criticado por estos grupos bajo el argumento de que podía ser utilizado "psicológicamente" como un mecanismo informal para disuadir el ejercicio al voto (Corrales y Penfold, 2007).

Incluso algunos sectores de la oposición afirmaron que este instrumento violaba el secreto del voto y que a través de este tipo de tecnología se podía identificar electrónicamente por cuál candidato había decidido votar el elector. Este tipo de argumentaciones, sustentadas en las fallas de una prueba del sistema automatizado realizado por el CNE con la asistencia de todos los partidos políticos antes de la realización de las elecciones legislativas de 2005, llevaron a la oposición a retirarse de la contienda electoral. No obstante, auditorías posteriores realizadas por la OEA no encontraron evidencias sobre la posibilidad de este tipo de resultados e hicieron recomendaciones al CNE para mejorar su uso en las elecciones presidenciales de 2006. Es preciso destacar que la oposición se retiró a pesar de que el CNE había aceptado remover los dispositivos dactilares de algunas circunscripciones electorales y a pesar de algunos acuerdos políticos que se habían alcanzado gracias a la facilitación de las misiones de la Unión Europea y la Organización de Estados Americanos (OEA).

En la actualidad el problema más relevante de la democracia electoral en Venezuela pareciera ser el uso de medidas administrativas por parte de la Contraloría General de la República, validadas por el CNE, para excluir a candidatos opositores y oficialistas de las elecciones (Petkoff, 2008). Para las elecciones regionales y locales de noviembre de 2008, la Contraloría General de la República inhabilitó a más de 300 candidatos, de los cuales 200 eran pertenecientes a la oposición. Todo ello, a pesar de que para esa fecha el oficialismo controlaba 22 de las 24 gobernaciones y más del $90 \%$ de las alcaldías. Las inhabilitaciones estaban fundamentadas en supuestas irregularidades administrativas que no habían sido validadas por los tribunales. Este precedente le otorgó a la Contraloría un poder excesivo para determinar cuáles candidatos podían ser inhabilitados sin que los casos hubiesen sido procesados por el sistema judicial. El efecto es la reducción de la competencia electoral y la violación de derechos políticos de diversos candidatos y sus partidos.

Otra falla que afecta el desempeño de la democracia venezolana en cuanto a su transparencia está vinculada a la falta de control del CNE sobre el uso de los recursos públicos por parte del oficialismo. Esta situación afecta la transparencia y equilibrio de las elecciones, no de manera directa sino a partir de desventajas notorias en la asimetría de financiamiento entre el oficialismo y la oposición para atraer el voto popular. Esta práctica viola la Constitución Bolivariana que establece que el financiamiento de los 
partidos políticos y sus actividades electorales no pueden tener origen público sino privado. El acceso irrestricto del movimiento oficialista al erario público, debido a la débil supervisión del CNE y del brazo legislativo, ha permitido que el "ventajismo" sea excesivo. Varios informes realizados por observadores internacionales durante los procesos electorales realizados en Venezuela durante los últimos cuatro años confirman esta opinión (Misión de Observación Electoral de la Unión Europea, 2007). Esta situación se ha complicado aún más con la falta de transparencia en el uso de los recursos petroleros provenientes de Petróleos de Venezuela S.A. (PDVSA) y su explícito uso clientelar en tiempos electorales. Diversos estudios han mostrado cómo en momentos electorales estos recursos han sido utilizados para "comprar votos" en una escala significativa y cómo los recursos de diversos programas sociales son asignados con criterios clientelares (Penfold, 2007; Rodríguez et al., 2008).

Finalmente, la Asamblea Nacional realizó en 2009 modificaciones sustantivas a la Ley Electoral facilitando la posibilidad de mitigar el efecto proporcional del sistema y ampliando la sobrerrepresentación de las mayorías. Para ello la nueva ley le dio autoridad al CNE para realizar procesos de reingeniería geográfica de las circunscripciones electorales (gerrymandering) sin ningún tipo de aprobaciones previas o posteriores y legaliza el uso de tarjetas simultáneas (llamadas en Venezuela "las morochas") que permiten a un mismo partido (actuando a través de un partido espejo) presentar listas idénticas para una misma circunscripción. Este efecto permite que los candidatos de un mismo partido puedan copar los puestos por listas y por circunscripción uninominal sin importar que sean presentados por partidos nominalmente diferentes pero exactos en cuanto a sus candidatos. De esta forma se hace un "crowding out" de los partidos minoritarios.

\section{b. La democracia constitucional}

El progresivo debilitamiento de la división de poderes dentro de la democracia venezolana es sin duda su mayor debilidad y la causa de una mayor erosión de las bases institucionales del sistema político. El Poder Ejecutivo tiene un claro dominio sobre los poderes Legislativo y Judicial. Este dominio se extiende sobre otros poderes como el Consejo Nacional Electoral, la Contraloría y la Fiscalía General de la República. Debilidades en el diseño constitucional del presidencialismo venezolano, junto con la posición hegemónica del oficialismo en el órgano legislativo, han incrementado los poderes formales e informales del Presidente de la República.

No hay duda que el dominio del partido oficialista en la Asamblea Nacional fue reforzado por la retirada de la oposición de las elecciones legislativas de 2005. Esta decisión por parte de la oposición le permitió al oficialismo obtener casi la totalidad de los puestos de representación. En últimas fechas han surgido algunas voces disidentes, producto de divisiones internas del chavismo, en particular dentro del partido Podemos y de Patria Para Todos. En este sentido, la falta de controles horizontales tiene orígenes que no son exclusivamente constitucionales sino también electorales y políticos. 
Esta ventaja del chavismo ha sido utilizada para minar los cimientos de la división de poderes e incrementar el control político sobre diversas instituciones. La conformación del Tribunal Supremo de Justicia fue ampliada en 2004 de tal manera que garantizó el dominio absoluto del Presidente de la República sobre las decisiones del Poder Judicial. Asimismo, la designación del Consejo Nacional Electoral en el año 2006 ha reflejado criterios políticos (Kornblith, 2007). El reciente nombramiento de la Fiscalía General de la República en el año 2007 mostró procedimientos similares. Tanto los procesos de nominaciones como de selección muestran la intervención de filtros políticos, mientras que la prevalencia del chavismo en la Asamblea Nacional hace creíble la posibilidad de sanciones en contra de estos funcionarios por decisiones que sean consideradas desfavorables por el Poder Ejecutivo.

La discusión sobre la reforma constitucional de 2007 y la propuesta de enmienda constitucional para eliminar los límites de reelección a la presidencia en 2008 evidenciaron la falta de controles horizontales y el dominio del Ejecutivo sobre el resto de los poderes. La propuesta de reforma constitucional partió del Ejecutivo en un discurso presidencial semanas después de la reelección de Chávez en 2006. Fue aprobada para someterla a consulta popular por el Legislativo con cambios menores en menos de dos meses, a pesar de que la propuesta de reforma era tan profunda que prácticamente implicaba la creación de una nueva Constitución e incluso la convocatoria de una Asamblea Nacional Constituyente. Los procesos de consulta estuvieron circunscritos a simpatizantes del proceso político chavista. La reforma incluía cambios sustanciales en el concepto de propiedad privada, la eliminación de los límites a la reelección presidencial, la reconfiguración territorial de los gobiernos regionales, la eliminación de la autonomía del Banco Central de Venezuela, la elevación de los requisitos para la convocatoria de referéndums, entre otros. Esta reforma fue derrotada por un pequeño margen (51\% por el No contra 49\% por el Sí) en el referéndum de diciembre de 2007 y contribuyó a mantener la polarización política del país.

La propuesta de enmienda surgió de una forma muy similar a la reforma constitucional. Después de las elecciones regionales y locales de 2008, el presidente Chávez anunció que una enmienda constitucional sería sometida ante el Poder Legislativo para eliminar la prohibición de reelección para los puestos de elección popular. En solo unas semanas, la Asamblea Nacional había aprobado la discusión de la enmienda para someterla una vez más a aprobación a través de una consulta popular. En este sentido, las propuestas de cambio de las reglas del juego establecidas por la Constitución Bolivariana emergen del Poder Ejecutivo con poco debate, tanto político como ciudadano.

Por otra parte, la falta de controles por parte del Legislativo sobre los actos del Poder Ejecutivo en el ámbito administrativo es notoria, en particular en lo referente a los controles sobre los gastos presupuestarios y extrapresupuestarios. La Asamblea Nacional, por iniciativa del Ejecutivo, aprobó una serie de reformas a la Ley del Banco Central de Venezuela (BCV) para limitar la obligación de PDVSA de vender los recursos de las exportaciones petroleras al Banco Central. Antes, PDVSA estaba obligado a vender todas sus divisas al BCV, salvo lo necesario para pagar los compromisos de la corporación petrolera en el exterior. Sin embargo con estas reformas, PDVSA puede transferir recursos 
al Ejecutivo Nacional o incluso gastar de manera directa en la compra de bolívares para financiar sus diversos programas. Estas modificaciones crearon las condiciones legales para formalizar un gasto extrapresupuestario, ejecutado a través de diversos fondos públicos -entre ellos, el FONDEN- sin que instituciones como la Asamblea Nacional o el Banco Central de Venezuela pudiesen supervisar esos gastos. La Asamblea Nacional también ha mostrado una disposición muy débil a través de sus diversas comisiones, en particular la Comisión de Finanzas y la Comisión de Contraloría, para supervisar la ejecución del presupuesto que es formalmente aprobado cada año por el Legislativo.

Los controles verticales del poder, es decir, la capacidad de los arreglos federales establecidos en la Constitución para mantener ciertos balances sobre el poder nacional, también se han visto erosionados. Todos estos procesos políticos, caracterizados por la polarización y el posicionamiento hegemónico del Poder Ejecutivo, tuvieron un corolario similar en las esferas regional y local. Las transformaciones del proceso de descentralización estuvieron ancladas en tres factores que permitieron la reversión parcial de las reformas institucionales durante el período chavista: el esfuerzo del Presidente por desmontar la coordinación política entre los gobernadores que había caracterizado exitosamente a la descentralización durante los noventa; el cambio de la arquitectura fiscal para fortalecer, en medio de la bonanza petrolera, al Poder Ejecutivo por encima de los gobiernos regionales y locales; y el impulso de dinámicas de participación popular, como la creación de los Consejos Comunales, como instancias que compiten de manera directa con los gobernadores y alcaldes por recursos fiscales y por la legitimidad política en el proceso de toma de decisiones.

Es necesario acotar que la descentralización en Venezuela no fue por completo revertida debido a que Chávez no pudo impedir la elección directa de alcaldes y gobernadores garantizada por la Constitución. La descentralización sufrió en el plano administrativo y fiscal pero no pudo ser anulada en los ámbitos electoral y político. Chávez logró este resultado a pesar de que la misma Constitución generaba las condiciones jurídicas para profundizar la descentralización. El texto constitucional ordenaba de manera explícita la necesidad de elegir de manera directa a los alcaldes y gobernadores por un período de cuatro años con posibilidad de reelección; establecía la posibilidad de transferir competencias tributarias a los estados a través de la creación de la Hacienda Pública Regional; incorporaba la figura del Consejo Federal de Gobierno como instancia presidida por el Vicepresidente para tratar los temas relativos a las transferencias intergubernamentales, en particular, la creación de un Fondo de Compensación Interterritorial; asimismo, estipulaba una banda entre el 15 y el 20\% de los ingresos ordinarios para la trasferencia directa a los estados y municipios a través del Situado Constitucional. En principio, todos estos cambios no cerraban ni revertían el proceso de descentralización, más bien parecían abrir opciones de profundización. Sin embargo, la dinámica política del país, en especial el fortalecimiento del Presidente por medio del debilitamiento de los pesos y contrapesos institucionales, minaron esta posibilidad.

Una de las primeras decisiones de Chávez al ser electo como Presidente en 1998 fue desconocer la existencia de la Asociación Nacional de Gobernadores (ANG). Chávez nunca quiso reunirse de manera formal con los gobernadores a través de este mecanismo 
institucional, pues era evidente que darle este reconocimiento a la ANG debilitaría las posibilidades de concentrar poder con el afán de avanzar con el proyecto revolucionario. Después de las megaelecciones del año 2000 (año en el cual Chávez fue reelecto después de ser aprobada la nueva Constitución y en la cual su partido ganó 8 gobernaciones), el Presidente optó por crear una institución paralela a la que había surgido durante los noventa. Fue así como Chávez decidió auspiciar la Asociación de Gobernadores Bolivarianos en la cual sólo eran invitados aquellos gobernadores pertenecientes a la alianza chavista conformada por el Movimiento al Socialismo (MAS), el Movimiento Quinta República (MVR) y Patria Para Todos (PPT). La Asociación Nacional de Gobernadores pasó a ser entonces un foro de gobernadores de oposición y, debido a ello, encontró cada vez más difícil avanzar en la agenda descentralizadora sin el apoyo de los gobernadores simpatizantes del gobierno. Por este motivo comenzó a perder fuerza el esfuerzo coordinador como mecanismo de presión sobre el gobierno central.

Asimismo, Chávez no convocó con la frecuencia establecida por la Constitución a las reuniones del Consejo Federal de Gobierno para evadir estas instancias de discusión colectiva. El Presidente sólo convocó sesiones del Consejo Federal en un puñado de oportunidades en 2002, bajo circunstancias de alta polarización política y con la intención de dar señales de reconciliación que nunca fueron materializadas.

Chávez también comenzó a entender la importancia de crear incentivos que pudiesen inducir negociaciones uno-a-uno en vez de negociaciones entre el Presidente y los gobernadores en conjunto. Para ello, el Presidente comenzó a utilizar el Fondo Intergubernamental para la Descentralización como mecanismo de control, en el cual sólo se aprueban los proyectos de inversión a aquellos gobernadores y alcaldes cercanos al oficialismo o que no muestran ambiciones políticas más allá de su estado o municipio. Esta estrategia ha sido replicada en el ámbito presupuestario al castigar a los estados de oposición que tenían responsabilidades transferidas en materias de educación y salud, como Mérida, Carabobo, Yaracuy y Zulia. Además, Chávez comenzó a transferir más recursos a las guarniciones militares esparcidas por todo el país para que tuviesen responsabilidades en la implementación de los programas sociales del Plan Bolívar 2000. La falta de capacidades de estas guarniciones y los escándalos de corrupción evitaron que esta estrategia tuviese mayor efectividad. Sin embargo, el esfuerzo del Presidente estaba orientado a minar la capacidad de los gobernadores y alcaldes para actuar de forma colectiva. Los gobernadores, en particular los oficialistas, pero también algunos miembros de la oposición, pronto comprendieron que para asegurar los recursos necesarios para su gestión era necesario negociar uno-a-uno con el Ejecutivo en vez de entrar en una coalición a través de la Asociación Nacional de Gobernadores. Para ellos la estrategia de los noventa de organizarse de manera colectiva parecía políticamente arriesgada y no aseguraba el acceso a los recursos fiscales dado el creciente poder del Presidente. En un discurso en el Teatro de la Academia Militar, justo después de las elecciones regionales y del referéndum revocatorio de 2004, un Chávez triunfador les recordó a los gobernadores que estaban invitados al acto: "aunque no puedo destituir a alcaldes ni a gobernadores, sí puedo hacer otras cosas, ¿no? Quiero que sepan que en 
esta nueva etapa el que está conmigo, está conmigo, el que no está conmigo está contra mí" (Harnecker, 2004).

Desde el comienzo del período chavista, el gobierno condujo una agenda centralizadora acompañada de la pérdida de transparencia y el aumento en la violación de las reglas constitucionales que regulan la transferencia de recursos fiscales a los estados y municipios. En Venezuela, un elemento medular de la eficiencia en materia de descentralización lo constituye el tratamiento de la volatilidad de las finanzas públicas, asociada a la condición de país petrolero, para impedir la transmisión de su impacto a las finanzas de los estados y municipios. Además, la tendencia centralizadora en materia económica, política y administrativa de Venezuela fue reforzada a lo largo de los últimos años por el incremento de los recursos provenientes de la renta petrolera percibidos sobre todo por el gobierno central.

La descentralización venezolana ha tenido como referencia general una base legal en la Constitución. Por ejemplo, en la Constitución de 1961 se declaraba a la nación como un estado federal y se establecía la autonomía política de los estados y municipios. Este mismo texto contemplaba la descentralización administrativa a través del Situado Constitucional y las Asignaciones Económicas Especiales, mediante los cuales se garantizaban los recursos necesarios para que los estados y municipios ejecutaran sus funciones. Más tarde, en la Constitución de 1999 se ratifica la definición del Estado como una entidad federal descentralizada y se establecen las disposiciones y principios que deben regir la gestión fiscal a nivel nacional, estatal y municipal. Por tanto, la Constitución proporcionó un marco legal para la instrumentación de reformas y políticas públicas orientadas hacia la ordenación gradual del federalismo fiscal de una forma responsable y sostenible.

La Constitución complementó el marco legal de la descentralización con la incorporación de la Hacienda Pública Estatal, el Consejo Federal de Gobierno y el Fondo de Compensación Interterritorial. Asimismo, incorporó los temas de creación de consejos regionales y locales de planificación y el aumento de los poderes de las comunidades a través de la posibilidad de transferencias de servicios a los grupos vecinales organizados. La Constitución ratificó la idea de que las fuentes de ingreso de los estados y municipios se instrumentarían a través del Situado Constitucional que, en un principio en la Constitución de 1961, tuvo un máximo de 15\% de los ingresos ordinarios percibidos por el Gobierno Central; sin embargo, la Ley Orgánica de Descentralización, Delimitación y Transferencias de Competencias llevó este monto a un 20\% de los ingresos. Este porcentaje luego fue ratificado como un techo máximo en la Constitución de 1999. No obstante, su regla de reparto entre estados continuó tal como estaba establecida en la Constitución de 1961: un 30\% en partes iguales y el 70\% restante con base en la población de cada entidad. La Asamblea Constituyente también dejó intactas las transferencias por concepto del Impuesto al Valor Agregado y de regalías petroleras establecidas para los instrumentos legales del FIDES y de la Ley de Asignaciones Económicas Especiales (LAEE), respectivamente.

A partir del año 2002 comenzaron a surgir nuevas estructuras de gobierno local que se superponían a las existentes. En primer lugar, con el objeto de estimular la planificación 
de los servicios municipales y la participación de las comunidades organizadas, se aprobó la Ley de Consejos Locales de Planificación que, conjuntamente con la nueva Ley del Poder Público Municipal, afectó la autonomía en lo relativo al ámbito municipal y a la coordinación de las políticas públicas. No obstante, con la aprobación de la Ley Especial de los Consejos Comunales en 2006, la Ley de los Consejos Locales de Planificación quedó derogada. En un principio los Consejos Comunales surgieron a instancias de los Consejos Locales de Planificación Pública, pero la Asamblea Nacional les concedió vida propia mediante un instrumento jurídico, lo que les permitió recibir y administrar recursos públicos y convertirse en actores de la gestión local. Estas nuevas estructuras, a diferencia de los estados y municipios, son órganos del Poder Ejecutivo Nacional, al cual deben su reconocimiento jurídico y dependencia financiera. Conforme a la Ley de los Consejos Comunales, estas estructuras sociales cuentan con recursos financieros provenientes de las siguientes fuentes: LAEE, FIDES, transferencias presupuestarias del gobierno central, transferencia de los estados y municipios y aquellos recursos provenientes de la administración de los servicios públicos que le sean transferidos por el Estado, los generados por su actividad propia, donaciones y cualquier otra actividad financiera.

En correspondencia con este objetivo, Chávez procedió a la reforma de la ley del FIDES y de la LAEE para proveer a los Consejos Comunales de fuentes de financiamiento, decisión que redujo aún más la capacidad financiera de los estados y municipios. Con la reforma de la ley del FIDES cambiaron las proporciones del reparto de sus recursos a $42 \%$ para los estados, $28 \%$ para los municipios y $30 \%$ para los Consejos Comunales, con respecto a su incorporación en las leyes presupuestarias; y a 30\% para los estados, $20 \%$ para los municipios y 50\% para los Consejos Comunales cuando se produzcan excedentes en la recaudación del IVA. Tanto los estados como los municipios han sido afectados con la Ley Especial de los Consejos Comunales, sancionada en abril de 2006, según la cual se les confiere capacidad de gestión directa en materia de políticas públicas y en la ejecución de proyectos orientados a responder a las demandas de las comunidades.

Todos estos cambios institucionales tuvieron un impacto notable sobre el comportamiento de las finanzas públicas a nivel regional y local. Si observamos los ingresos del gobierno central durante la última década, estos muestran un comportamiento volátil debido a su alta dependencia en los ingresos petroleros. Como hemos visto, las leyes establecieron que las transferencias del gobierno central a los estados y municipios eran un porcentaje de los ingresos ordinarios estimados en el presupuesto para cada año. Sin embargo, el Presidente Chávez, con el fin de centralizar los recursos, subestimó de forma recurrente los ingresos en el presupuesto nacional, por lo que las transferencias legales fueron menores a lo que en realidad el gobierno debía transferir a los estados y municipios. De acuerdo con la vocación centralista y presidencialista del proyecto chavista, en la última década se utilizaron la subestimación de los ingresos fiscales petroleros y no petroleros en el presupuesto nacional y la demora en la entrega de los recursos como mecanismos de elusión de las asignaciones legales.

Las diferencias entre las transferencias efectivas y las que el gobierno debió transferir en función de su ingreso real presentaron una tendencia ascendente que se duplicó al pasar de un $20 \%$ en 1998 a un $40 \%$ en 2007. En consecuencia, los ingresos de las gobernaciones 
y alcaldías provenientes de las transferencias legales fueron cada vez menores. A finales de 2003, los precios del crudo comenzaron a subir y, como consecuencia, el Ejecutivo comenzó una fuerte expansión fiscal y la adopción del uso de fondos extrapresupuestarios para orientar el gasto a proyectos discrecionales. Estos gastos extrapresupuestarios -Fondo para el Desarrollo Económico y Social del País (FONDESPA), Fondo de Desarrollo Nacional (FONDEN) y Fondo Miranda- no estaban sujetos a control ni rendición de cuentas, eran utilizados a discreción por el Poder Ejecutivo y podían acumularse en cuentas externas.

Debido a estas circunstancias, a partir del año 2003 la diferencia entre las transferencias legales entregadas y las que debía haberse entregado a los estados y municipios se hizo cada vez más marcada, al pasar de un promedio para el período 1998-2002 de $24 \%$ a un promedio de $40 \%$ entre 2003 y 2007. Por otro lado, mientras que las transferencias legales para el período en estudio (1998-2007) permanecieron en niveles de 5\% del PIB, es muy difícil estimar cuánto debieron haber sido las transferencias que el gobierno debió trasladar a las regiones tomando en cuenta el gasto extrapresupuestario. El punto es sencillo: aunque se desconozca el monto real ejecutado, la evidente expansión del gasto extrapresupuestario del gobierno central en los últimos años no sólo reforzó la dirección centralista de las finanzas públicas, sino que reflejó un total desprecio hacia la descentralización, la transparencia y la rendición de cuentas. En general, la política fiscal implementada en los últimos años ha sido contraria a lo establecido en la Constitución en lo relativo a la conformación de un estado federal descentralizado y la ejecución de una gestión fiscal disciplinada basada en los principios de eficiencia, solvencia, transparencia, responsabilidad y equilibrio fiscal; así como de la instrumentación de un fondo de estabilización macroeconómica que realmente lograra estabilizar el gasto fiscal a nivel central, regional y municipal.

\section{c. La Democracia Ciudadana}

La dimensión ciudadana de la democracia implica la capacidad de los individuos para influir a través de deliberaciones públicas y desde un punto de vista electoral en las decisiones colectivas. En principio, la Constitución de 1999 incorpora diversas formas de participación ciudadana en las decisiones públicas. La primera de ellas es la consideración de referéndums (revocatorio, consultivo, aprobatorio) que comenzaron a ser integradas a finales de la década de los noventa con la reforma del sistema electoral. Asimismo, la Constitución establece la capacidad de los ciudadanos para activar de manera directa estos mecanismos de participación, aunque los mismos también pueden ser iniciados por los poderes Ejecutivo y Legislativo.

Hasta el momento, los venezolanos han podido utilizar dos de estos tipos de referéndums: el revocatorio y el aprobatorio. El referéndum revocatorio fue activado en contra de la figura del Presidente de la República en el año 2004 por medio de la iniciativa popular. El referéndum aprobatorio fue utilizado para la convocatoria de la Asamblea Constituyente y la aprobación de la Constitución en 1999, así como para evaluar la posibilidad de reformas a la Constitución en el año 2007. Sin embargo, es de hacer notar que los referéndums 
aprobatorios fueron activados por la Presidencia de la República o por la Asamblea Nacional. El referéndum consultivo intentó ser activado por iniciativa popular en el año 2003 para evaluar la gestión del Presidente de la República, pero su activación fue declarada nula por el CNE y por el Tribunal Supremo de Justicia.

El contraste entre democracia representativa y democracia participativa fue llevado por Chávez a la esfera territorial en la creación de los Consejos Comunales. Estas instancias de participación ciudadana fueron diseñadas en contraposición al poder formal de los gobernadores y alcaldes. El presidente Chávez justificó estos Consejos Comunales como un paso aún más radical que la descentralización al darle la posibilidad a las comunidades no sólo de identificar sus necesidades sino de administrar bienes y servicios públicos de manera directa. Sin duda la idea de la participación popular es un mecanismo necesario y fundamental en un sistema descentralizado y democrático para garantizar la adecuada identificación de necesidades, para determinar y monitorear el presupuesto municipal y para coordinar el control social en la gestión del gobierno. Sin embargo, en este caso, las nuevas instancias fueron vistas no como un complemento sino como un competidor directo de los diversos niveles de gobierno. Los Consejos Comunales fueron creados para competir y no para complementar y asegurar mejoras en la calidad de los gobiernos regionales y locales. Un buen ejemplo es que los mecanismos de asignación de recursos están atados al poder del Presidente y son independientes de la existencia de cooperación entre los alcaldes, gobernadores y dichos Consejos Comunales. Para el gobierno central, la gestión de los Consejos Comunales es autónoma de las acciones de los gobernadores y alcaldes. De esta forma se convierten en una vía para que el Poder Ejecutivo penetre en las comunidades más allá de la filiación política de las autoridades regionales.

El gobierno central prefirió favorecer un diseño institucional en el que los Consejos Comunales se multiplicaran de manera indiscriminada dentro de una misma unidad territorial. En los 335 municipios que existían a nivel nacional en el año 2008 se habían conformado más de 26.000 instancias de participación directa (Machado, 2009). El gran número de Consejos Comunales comenzó a generar dos tipos de problemas. En primer lugar, hacía difícil la cooperación entre ellos para enfrentar las demandas locales en coordinación con los gobiernos municipales, regionales e incluso con el gobierno central. Esta multiplicidad comenzó a generar una competencia por recursos escasos y muchas veces incentivó conflictos sociales innecesarios debido a que no eran claros los criterios de asignación de recursos. En segundo lugar, la gran cantidad de Consejos Comunales hacía muy costosa la supervisión de los diversos proyectos, por lo que se abría una oportunidad muy grande para prácticas de corrupción.

La abundante renta petrolera le permitió al gobierno central comenzar a utilizar diversos fondos públicos con el objetivo de financiar estos Consejos Comunales. La falta de transparencia en los criterios de transferencias y su gestión sin una visión técnica de la viabilidad de los diversos proyectos permitirían que el clientelismo político se apoderase de la asignación de los recursos. Al igual que las misiones, los Consejos Comunales comenzarán a formar parte del andamiaje clientelar del aparato estatal (Machado, 2009). Su creación habrá generado una contradicción al representar de manera simultánea un 
mecanismo de empoderamiento popular descentralizado y una forma de control fiscal y político impuesto desde el centro.

\section{DERECHOS SOCIALES Y PARTICIPACIÓN POPULAR}

Más allá de las valoraciones ideológicas que se le puedan dar a la Revolución Bolivariana es evidente que este proceso político ha logrado centrar buena parte del debate sobre políticas públicas alrededor de la reivindicación de los derechos sociales y la activación de la participación popular como mecanismo de instrumentación de estos derechos. A pesar de que en Venezuela la participación popular ha sido un instrumento de movilización en el pasado, particularmente durante la década de los cincuenta y sesenta, no fue sino hasta el surgimiento del fenómeno chavista, a comienzos del milenio, que la promoción de mecanismos organizativos de base (como los Consejos Comunales, las Mesas Técnicas de Agua y Telecomunicaciones, las Cooperativas, entre otras) volvió a tener un papel preponderante en el intento de recrear una nueva relación entre el Estado y la sociedad.

Asimismo, la Constitución Bolivariana de 1999 realizó una expansión significativa de derechos sociales estableciendo explícitamente mecanismos de participación popular para su concreción (p.e. los consejos de planificación local). En el campo de las políticas públicas, Chávez diseñó una serie de programas sociales, conocidos como misiones, de corte fundamentalmente asistencialista, para garantizar a las capas más pobres acceso básico a una amplia gama de servicios sociales (Penfold, 2007). El gobierno también intentó fortalecer patrimonialmente el sistema de Seguridad Social para garantizar el pago de pensiones a personas de la tercera edad (Weisbrot et al., 2009). Finalmente, Chávez implementó a través de los Consejos Comunales y las Mesas Técnicas de Aguas y las Mesas Técnicas de Telecomunicaciones mecanismos de participación para levantar información sobre las necesidades de acceso a diversos servicios (especialmente agua y telecomunicaciones) por parte de sectores populares y rurales (Machado, 2009; Arconada, 2006). Este esfuerzo ha sido sin duda una fuente de apoyo político efectivo tanto real como simbólico, lo cual ha amplificado la popularidad y el discurso inclusionista del chavismo.

Sin importar la técnica estadística utilizada para medir el índice de pobreza, estos números han mostrado un descenso significativo, particularmente entre los años 20032007, un período de tiempo marcado por alto ingresos petroleros (Weisbrot et al., 2009; España, 2007). Para el año 2003, según cifras oficiales, el 61,0\% de la población vivía en una posición de pobreza. En el año 2007, tan sólo el 35\% de la población vivía en estas condiciones (España, 2007). Sin embargo, existe un amplio debate sobre si dados los altos ingresos petroleros estos indicadores debieron haber descendido aún más rápido (Rodríguez, 2008b) y sobre el sustrato de la calidad y sustentabilidad de las misiones (España, 2007; Penfold, 2007). Algunos estudios especializados en el tema de pobreza en Venezuela muestran la alta penetración de estos programas sociales. Por ejemplo, la Misión Mercal (de distribución de alimentos) alcanzó en el año 2007 hasta el 47,2\% de la 
población; la Misión Barrio Adentro (Salud Asistencial) a más del 25\% de la población; y la Misión Ribas (Educación Secundaria) a un 5,7\% de beneficiarios (España, 2007).

En el área de servicios básicos, como por ejemplo agua y telecomunicaciones, el esfuerzo fue igualmente importante. En el período 2004-2007 se instalaron más de 1.500 mesas técnicas de agua y telecomunicaciones en todo el país. Estas mesas estaban orientadas a concertar estrategias de acceso y mejora de la calidad de servicios públicos entre las comunidades y las compañías públicas (Arconada, 2006). Como se mencionó anteriormente, el gobierno también promovió la creación de Consejos Comunales para orientar inversiones públicas directamente en la comunidad a través de un modelo participativo. Para marzo de 2008, en Venezuela existían más de 36.812 Consejos Comunales; de este número tan sólo 26.143 estaban formalmente constituidos, mientras que el restante estaba en proceso de formación (Machado, 2009). En su gran mayoría los proyectos implementados por los Consejos Comunales estaban orientados a proyectos de infraestructura pública, urbanismo y servicios. En una encuesta realizada por la Fundación Gumilla, los beneficiarios respondían que un 23\% invertía en vivienda, 21\% en agua potable y servidas, $15 \%$ en vialidad, $14 \%$ en electrificación, $13 \%$ en deporte, entre otros (Machado, 2009). El gobierno también promovió, a través de subsidios directos, la creación de estructuras cooperativas. En un período relativamente corto, Venezuela pasó de tener poco más de 30.000 cooperativas a registrar formalmente más de 190.000 .

Evidentemente estos mecanismos de organización popular permitieron una expansión significativa en el acceso por parte de la población más pobre a diversos derechos sociales. Sin embargo, también existe evidencia que estos mismos mecanismos fueron utilizados clientelarmente. Además de ello, algunos de estos programas, en especial las misiones, precisamente porque fueron politizados, han perdido sustentabilidad. Diversos estudios han mostrado cómo las misiones fueron dirigidas a sectores de bajos ingresos no sólo con un objetivo redistributivo sino también para la compra directa de votos (Penfold, 2007; Penfold y Ortega, 2008). Adicionalmente, alguno de estos programas, como por ejemplo, Barrio Adentro (Salud) prácticamente ha colapsado por falta de inversión pública y corrupción. Las Mesas Técnicas de Agua y Telecomunicaciones también han sido objeto de críticas en el sentido de que la discusión comunitaria está profundamente distorsionada por criterios ideológicos y políticos. Los mismos ciudadanos que participan directamente en los Consejos Comunales denuncian la presencia de la corrupción como un elemento que disminuye la efectividad en la concreción de los proyectos (Machado, 2009). Y finalmente, el gobierno nacional tuvo que desmontar los subsidios directos a las cooperativas, pues muchas de estas eran formalizaciones falsas para acceder a créditos blandos.

\section{EL HIPERPRESIDENCIALISMO VENEZOLANO}

Venezuela es percibida, cada vez con más frecuencia, como una democracia poco consolidada a pesar de su larga tradición en el ejercicio del derecho al voto y su esfuerzo 
por ampliar la base de derechos sociales. Parte del problema se refiere a la inestabilidad política, los profundos cambios político-constitucionales y la pérdida de autonomía de los poderes públicos. Además, las instituciones democráticas no son reconocidas como espacios neutrales para la participación ciudadana y la resolución de conflictos políticos, sociales y electorales.

Los sistemas democráticos se consideran consolidados cuando la incertidumbre política se encuentra institucionalizada (Przeworski, 1991; Weingast, 1997). No se puede hablar de democracia si los resultados son anticipados con plena certeza antes de una elección, pues en ese caso se trataría de una autocracia en la que todo es determinado ex ante (Przeworski, 1991). Esto quiere decir que la incertidumbre es una característica intrínseca del buen funcionamiento de cualquier democracia. Esta incertidumbre con respecto a los resultados electorales motiva a los distintos actores a participar en los comicios, pues se tiene la certeza de que existe alguna probabilidad de ganar u obtener algún tipo de representación política. Cuando esta incertidumbre ha sido institucionalizada -las reglas que regulan la competencia electoral y la división de poderes son aceptadas por todos los actores políticos sin importar los resultados de la contienda electoral-, el sistema democrático se encuentra en equilibrio y puede ser considerado estable. Otros factores esenciales para considerar un sistema democrático consolidado incluyen el control civil sobre las fuerzas armadas, así como la existencia de libertades civiles y políticas protegidas por el estado de derecho (Linz y Stepan, 1996). Sin la existencia de estas condiciones cualquier democracia puede experimentar serias barreras que ponen en peligro su propia supervivencia.

$\mathrm{Al}$ analizar el estado de la democracia en Venezuela desde esta perspectiva es posible argumentar que la fuente de inestabilidad de los últimos años tiene un origen institucional. Las características propias de la Constitución de 1999, que otorga excesivos poderes al Presidente de la República, y la falta de división de poderes crean dificultades para que los distintos actores del sistema político acepten las reglas de juego en el marco de un sistema de partidos débil y poco estructurado.

¿Cuáles son los factores que por diseño constitucional elevan los costos de perder en las elecciones? El primero es la introducción de un período presidencial de seis años con reelección. La posibilidad de un período continuo de 12 años es sin duda el más largo de América Latina y la eliminación de restricciones a la reelección hace este problema aún más agudo. En segundo lugar, para el Presidente de la República es relativamente sencillo convocar distintos tipos de referéndum para modificar de manera unilateral las reglas del juego, incluyendo la propia Constitución. En tercer lugar, la Constitución le otorga poderes importantes al Presidente en la administración de las fuerzas armadas, en particular para determinar los ascensos. Por último, la Constitución debilitó los poderes de la Asamblea Nacional al reducir su ámbito de acción en el manejo de los temas federales y en la supervisión de las fuerzas armadas. Esto fue posible debido a la eliminación de la Cámara del Senado y la adopción de un sistema unicameral.

El presidencialismo establecido en las instituciones formales de Venezuela se ha visto exacerbado en el plano informal gracias a la falta de controles sobre el gasto de los 
ingresos petroleros. La existencia de fondos parafiscales alimentados desde PDVSA y el Banco Central de Venezuela, sin controles previos por parte de la Asamblea Nacional, le permite al Poder Ejecutivo contar con recursos extraordinarios para financiar diversos proyectos. Durante el período 2004-2008 estos fondos parafiscales llegaron a manejar montos similares a los establecidos en el presupuesto formal. Buena parte de estos recursos fueron utilizados en proyectos sociales y de infraestructura que no obedecían a criterios técnicos sino a consideraciones clientelares.

Por si fuera poco, el Presidente Chávez ha realizado esfuerzos importantes para fortalecer aún más el presidencialismo: primero, a través de la reforma fallida de la Constitución $y$, posteriormente, por medio de la enmienda constitucional orientada a eliminar las restricciones para la reelección a cualquier puesto de elección popular. De esta manera el Presidente Chávez ha logrado consolidar el sistema más presidencialista de la región.

Como se mencionó en párrafos anteriores, la Constitución venezolana ya garantizaba el período más largo de cualquier presidente latinoamericano (12 años en caso de ser reelecto) y con la modificación del texto constitucional este período se vuelve indefinido (algo inexistente en otras constituciones presidencialistas en América Latina y Asia). Las elecciones al final de cada período presidencial y la posibilidad de un referéndum revocatorio se vuelven en las únicas restricciones institucionales a plazos indefinidos para administraciones presidenciales. Este cambio incrementa de manera considerable el poder del Presidente en un contexto en el que la división de poderes en Venezuela ya era casi inexistente.

Los resultados del referéndum de 2009 mostraron un fuerte repunte de la popularidad del presidente Chávez, quien logró conseguir $54,36 \%$ de los votos para el SÍ, frente a una oposición que lució sin liderazgo político al obtener $45,63 \%$ de los votos para el NO. Esta diferencia porcentual de casi 10 puntos le permite al Presidente legitimar de nuevo su mandato, evitar la posible activación de un referéndum revocatorio, consolidar el control y cohesión de su movimiento partidista para las elecciones legislativas de 2010 y frenar los avances electorales que la oposición había obtenido al derrotar la reforma constitucional en 2007 y obtener las gobernaciones de los estados urbanos en 2008.

El triunfo del SÍ no sólo tiene consecuencias constitucionales sino que, desde un punto de vista político, coloca un freno al deterioro que comenzaba a mostrar el chavismo y obliga a la oposición a replantear su estrategia electoral. En este sentido, el triunfo del Sí tiene consecuencias fundamentales para el presidente Chávez al garantizar su estabilidad política en medio de una situación económica compleja y detener un sensible avance electoral que comenzaba a mostrar el sector opositor.

La decisión del presidente Chávez de impulsar la enmienda constitucional para garantizar su reelección indefinida obedeció a razones fundamentalmente políticas. La derrota de la reforma constitucional por un margen pequeño en 2007 (resultados aún no oficializados por el CNE) representó la primera derrota de un proyecto político cuya ambición estaba orientada a redefinir el concepto de propiedad privada, a rediseñar la arquitectura territorial y continuar fortaleciendo los poderes presidenciales a través de la reelección continua del Poder Ejecutivo. Este revés mostró los límites ideológicos a los que podía 
llegar el elector venezolano y encontró muy poco eco entre los líderes chavistas a nivel regional y local para movilizar el voto en esta dirección.

Las elecciones para gobernadores y alcaldes en el 2008 también mostraron grietas importantes dentro del chavismo, así como avances sustanciales en el mundo opositor. La consolidación del Partido Socialista Unido de Venezuela (PSUV) implicó el abandono de una coalición con partidos minoritarios (PODEMOS, PPT, Partido Comunista de Venezuela) que al chavismo le había garantizado triunfos muy contundentes desde el año 2004. La decisión de Chávez de radicalizar el proyecto a través del impulso a la reforma constitucional y el abandono de esta coalición partidista tuvo costos electorales importantes que significaron su derrota posterior en el año 2007. Incluso, las divisiones internas del PSUV se hicieron evidentes en el proceso de las elecciones primarias para seleccionar a los candidatos a alcaldes y gobernadores y obligaron al presidente Chávez a intervenir en casos puntuales para imponer ciertos líderes partidistas.

En términos cuantitativos el presidente Chávez había logrado obtener casi el 52\% de los votos para las elecciones regionales y locales de 2008 a través de la obtención de 18 de las 23 gobernaciones y más del $80 \%$ de las alcaldías. Sin embargo, el haber perdido en 5 de las gobernaciones de mayor importancia (Miranda, Carabobo, Zulia, Nueva Esparta y Táchira) y centros urbanos y populares (Alcaldía Sucre y Alcaldía Mayor) lo obligaron a buscar una enmienda constitucional que garantizara la estabilidad de su liderazgo. Este hecho se vio agravado por la derrota sufrida por otros líderes de su movimiento (Diosdado Cabello y Aristóbulo Isturiz) en estos mismos comicios.

Para superar esta desventaja política, Chávez propuso una enmienda que en principio tan sólo eliminaba las restricciones para la reelección del Poder Ejecutivo. Esta misma propuesta había sido derrotada en la reforma constitucional de 2007 y no contaba con el apoyo de la opinión pública. Casi el 66\% de la población rechazaba la idea de reelección indefinida para el Presidente y cerca de un tercio de los chavistas no compartían esta iniciativa. Esta situación obligó al presidente Chávez a modificar la enmienda e incluir a todos los puestos de elección popular tales como gobernadores y alcaldes. Este cambio logró alinear a su base política con el liderazgo del presidente Chávez para avanzar con una campaña que contó con acceso prácticamente ilimitado a los recursos públicos.

El Consejo Nacional Electoral (CNE) también contribuyó políticamente al facilitar la convocatoria del proceso y abstenerse prácticamente de monitorear la campaña informativa. La presidenta del CNE, Tibisay Lucena, accedió a organizar el proceso refrendario sin que estuviese formalmente aprobado por parte del Poder Legislativo, con el objetivo de acortar los lapsos preparativos para la consulta popular. Esta decisión permitió evitar las consecuencias desfavorables que tenía para la popularidad del presidente Chávez la desaceleración económica como consecuencia de la caída de los precios petroleros. Esta decisión extemporánea incluso obligó al CNE a reimprimir el material electoral una vez que el Poder Legislativo decidió, por solicitud del presidente Chávez, incluir al resto de los cargos de elección popular para la enmienda constitucional.

Además de ello, el CNE tomó una serie de decisiones que aumentaron significativamente la asimetría de poder entre el oficialismo y la oposición. Primero, para contribuir con 
la reducción de los lapsos de preparación del referéndum, el CNE decidió no reabrir el registro electoral. Esta decisión era inconsistente con la jurisprudencia venezolana y con la práctica de este mismo CNE de aumentar la inclusión de nuevos electores en el registro electoral tal como lo había hecho en comicios anteriores. Segundo, el CNE decidió no financiar con dinero público la campaña de información tanto del Sí como del NO. En efecto, la Constitución Bolivariana prohíbe el uso del financiamiento público para la realización de campañas electorales. Sin embargo, un referéndum no es una elección sino una consulta popular. El mismo CNE en el año 2007 había tomado la decisión, acertada desde un punto de vista democrático, de financiar las campañas de información tanto del SÍ como del NO para la reforma constitucional. En esta nueva oportunidad, el CNE decidió complacer al oficialismo y limitar el acceso a este tipo de financiamiento. Las consecuencias fueron terribles para una oposición que dependía prácticamente de forma exclusiva de un financiamiento privado y que venía de unas elecciones regionales y locales extremadamente costosas. Por último, el CNE decidió no limitar ni castigar el uso de los recursos del Estado por parte del oficialismo, lo cual produjo una campaña dominada por la proyección del Sí. Esta diferencia en cuanto al músculo financiero de las campañas tuvo un impacto determinante en el ejercicio del voto.

Los medios de comunicación de alcance nacional y cuasinacional, en particular Venezolana de Televisión, Tves y Globovisión, agravaron esta situación y mostraron un desempeño poco ético y parcializado. Estas frecuencias televisivas fueron utilizadas con motivaciones partidistas durante la campaña, mostrando uno de los peores rostros de la política y los medios de comunicación venezolanos. En cambio, canales como Televen y Venevisión mostraron un mayor equilibrio informativo, aunque en ocasiones muestran claros rasgos de autocensura.

El equilibrio en la cobertura de los eventos políticos en Venezuela continúa como uno de los elementos más complejos de resolver, pues se encuentra relacionado con la polarización política y la falta de financiamiento público de los partidos políticos, quienes dependen del acceso a medios parcializados para su proyección nacional. La fortaleza financiera y regulatoria del oficialismo, debido a su amplio acceso a los recursos del Estado, indujo un comportamiento disuasivo incluso en aquellos medios que intentaron ser más imparciales. Todo ello en un contexto en el que el Estado venezolano ha dejado de renovar licencias televisivas sobre la base de argumentos políticos (caso RCTV).

Los resultados electorales no sorprendieron. El presidente Chávez logró aumentar su votación porcentual en el referéndum para la enmienda, en comparación con las elecciones de gobernadores y alcaldes de 2008, en poco más de 2 puntos. En términos generales, aquellos estados que habían votado por el oficialismo en las elecciones de 2008 intensificaron sus preferencias chavistas en el referéndum (por ejemplo, Monagas, Portuguesa, Guárico, Barinas, Trujillo, Cojedes, Aragua y Bolívar). Por el contrario, los estados que habían votado por las fuerzas opositoras mantuvieron su posición sin mostrar mayor crecimiento en términos relativos durante el referéndum (Miranda y Zulia). En algunos estados de oposición se logró intensificar el voto antichavista (Táchira), mientras que en otros estados dominados por la oposición (Nueva Esparta y Carabobo) 
el chavismo logró aumentar su votación en términos relativos. Esta intensificación del voto chavista en aquellos territorios consolidados y la falta de crecimiento de la oposición fue lo que determinó el triunfo electoral del SÍ durante el proceso de referéndum para la enmienda constitucional.

Venezuela se adentra en un proceso institucional muy frágil que, aunque no cancelará su democracia electoral, continuará mermando la división de los poderes constitucionales. La enmienda de 2009 implica la consolidación de un sistema hiperpresidencialista y de una serie de caudillos regionales (tanto de oposición como oficialistas) que convertirán a la reelección indefinida en un instrumento de poder para cohesionar sus diversos movimientos políticos y sociales. La consecuencia de esta tendencia es el debilitamiento permanente de instituciones intermedias de carácter representativo (Poder Legislativo) e incluso de aquellas instancias de resolución de conflictos (Poder Judicial y Electoral).

\section{CONCLUSIONES}

Venezuela posee una democracia de corte únicamente electoral cercada y cada vez más subyugada por los poderes presidenciales. Desde un punto de vista formal, la democracia constitucional y ciudadana se vuelven limitadas debido al continuo debilitamiento de la división de poderes. En sentido estricto, la democracia venezolana está cada vez más restringida al ámbito electoral a pesar de una ampliación de ciertos derechos sociales. Sin embargo, los últimos comicios comienzan a mostrar signos preocupantes que podrían afectar incluso el ámbito electoral de la democracia. La falta de autonomía del Consejo Nacional Electoral crea asimetrías significativas que aumentan las inequidades en la competencia política. Estas inequidades son cada vez más grandes, en especial en un contexto en el que el Presidente y su movimiento político controlan el acceso a la renta petrolera y a las instituciones establecidas. Aún más preocupante para la democracia electoral es el uso de "inhabilitaciones administrativas" orientadas a penalizar el uso de los derechos políticos. Este tipo de prácticas le permite al oficialismo excluir a potenciales candidatos opositores de la arena electoral y escoger así a sus contrincantes. El uso continuo de estas medidas podría socavar también la dimensión electoral del sistema político venezolano.

\section{REFERENCIAS}

Arconada, Santiago. 2006. Mesas Técnicas de Aguas y Consejos Comunitarios de Agua. Revista Venezolana de Economía y Ciencias Sociales 12 (2): 127-132.

Centro de Auditoría y Promoción Electoral (CAPEL). 2005. “Informe Técnico Auditoría Internacional del Registro Electoral República Bolivariana de Venezuela", Costa Rica.

Corrales, Javier y Michael Penfold. 2007. "Venezuela: Crowding Out the Opposition", Journal of Democracy 18 (2): 99-113.

Corrales, Javier. 2006. "Hugo Boss". Foreign Policy 152: 32-40.

Dahl, Robert: Polyarchy: Participation and Opposition. New Haven: Yale University Press.

Diamond, Larry y Leonardo Morlino. 2004. "The Quality of Democracy. An Overview". Journal of Democracy 15(4): 20-31. 
España, Luis Pedro. 2007. Programas Sociales y Condiciones de Vida en Venezuela 1999-2007. Center For Strategic and International Studies. Washington D.C.

Fernandes, Sujatha. 2007. "A View from the Barrios: Hugo Chávez as an Expression of Urban Popular Movements", LASA Forum 38 (1): 17-19.

Harnecker, Martha. 2004. “Comprender la Revolución Bolivariana: Martha Harnecker conversa con Hugo Chávez". Caracas: Centro Internacional Miranda.

Kornblith, Miriam. 2007. "Venezuela: Calidad de las Elecciones y Calidad de la Democracia". América Latina Hoy 45 (1): 109-124.

Linz, Juan y Alfred Stepan. 1996. Problems of Democratic Transition and Consolidation. Princeton: Princeton University Press.

Lupi, Juan Pablo y Leonardo Vivas. 2005. "(Mis)Understanding Chávez and Venezuela in Times of Revolution". The Fletcher Forum of World Affairs 29 (1): 81-102.

Machado, Jesús. 2009. Participación Social y Consejos Comunales en Venezuela. Revista Venezolana de Economía y Ciencias Sociales 15 (1): 173-185.

Mainwaring, Scott, et al., 2001. "Classifying Political Regimes in Latin America, 1945-1999". Studies in Comparative International Development 36 (1): 37-65.

Misión de Observación Electoral de la Unión Europea. 2006. "Informe Final. Elecciones Presidenciales Venezuela 2006". Bruselas: Unión Europea.

O'Donnell, Guillermo, et al., 2004. The Quality of Democracy. Theory and Applications. Notre Dame: Notre Dame University Press.

Ottaway, Marina. 2003. Democracy Challangeo: The Rise of Semi-Authoritarianism. Washington, D.C.: Carnegie Press.

Penfold, Michael. 2007. "Clientelism and Social Funds: Evidence from Chávez's Misiones Programs". Latin American Politics \& Society, Vol. 49 (4): 63-84.

Penfold, Michael y Daniel Ortega. 2008. "El Retorno Electoral de las Misiones". Caracas: Instituto de Estudios Superiores de Administración, Papel de Trabajo.

Petkoff, Teodoro. 2008. "A Watershed Moment in Venezuela". Interamerican Dialogue (documento de trabajo).

Przeworski, Adam. 1991. Democracy and the Market. Cambridge: Cambridge University Press.

Rodríguez, Francisco, et al., 2008. "The Price of Political Opposition: Evidence from Venezuela's Maisanta". Social Science Research Network (documento de trabajo).

Rodríguez, Francisco. 2008. "An Empty Revolution". Foreign Affairs March/April.

Weingast, Barry. 1997. "The Political Foundation of Democracy and the Rule of Law". American Political Science Review 91 (2): 245-264.

Weisbrot, Mark y Luis Sandoval. 2007. "The Venezuelan Economy in the Chávez Years". Washington DC: Center for Economic and Policy Research.

Weisbrot, Mark and Rebecca Ray y Luis Sandoval. 2007. "The Chávez Administration at 10 Years: The Economy and Social Indicators". Washington DC Center for Economic and Policy Research February 2009.

Michael Penfold es Profesor Asociado del Instituto de Estudios Superiores de Administración (IESA) en Caracas, Venezuela. Posee un Ph.D. de la Universidad de Columbia en Nueva York especializándose en temas sobre política comparada y economía política. Ha sido Profesor Invitado de la Universidad de Columbia, Universidad de los Andes en Bogotá, Universidad del Sur de California, y Sciences Po en París. Es autor y editor de diversos libros, entre ellos el más reciente, Dos Tradiciones, Un Conflicto: El Futuro de la Descentralización (Random House Mondadori, 2009). Ha publicado en diversas revistas académicas internacionales como el Journal of Democracy, Latin American Politics and Society, Americas Quarterly, Harvard Business Review América Latina y Lasa Forum, entre otras. Ha sido premiado por la Tinker Foundation y la Fundación Ford y ha sido becario de la Fundación Fulbright. (E-mail: Michael. penfold@iesa.edu.ve). Tlf: 5554210. 